NONCOMMUTATIVE HARMONIC ANALYSIS

WITH APPLICATIONS TO PROBABILITY III

BANACH CENTER PUBLICATIONS, VOLUME 96

INSTITUTE OF MATHEMATICS

POLISH ACADEMY OF SCIENCES

WARSZAWA 2012

\title{
WEHRL ENTROPY OF THE STATE IN A TWO-ATOM TAVIS-CUMMINGS MODEL
}

\author{
DEBRAJ NATH \\ Department of Mathematics, Vivekananda College \\ 269, Diamond Harbour Road, Kolkata-700063 \\ E-mail:debrajn@gmail.com \\ P. K. DAS \\ Physics and Applied Mathematics Unit, Indian Statistical Institute \\ 203, B. T. Road, Kolkata-700108 \\ E-mail:daspk@isical.ac.in
}

\begin{abstract}
In this paper we present an entropic description of quantum state obtained by interaction of one mode of quantized electromagnetic field with two two-level atoms inside a cavity, known as Tavis-Cumming model. Wehrl entropy has been calculated analytically and investigated as a function of the average value of the photon number operator. Husimi's $Q$ function has been calculated and compared with the behaviour of the field entropy.
\end{abstract}

1. Introduction. An interesting fundamental theoretical model of the interaction between two quantum systems is Tavis-Cummings model (TCM) 21] which describes the interaction of a single mode of quantized electromagnetic field with an ensemble consisting of two two-level atoms. Within the rotating wave approximation and under certain initial conditions this is an exactly solvable model and the understanding of the dynamical evolution of the TCM may throw some light on the performance of quantum information processing which in its turn may help the understanding of fundamental quantum mechanics. The one atom case which is the well known Jaynes-Cummings model and can be considered as a bipartite entanglement system has been widely investigated for initial pure states and mixed states of the field. The correlation that develops between the atom and the field during the interaction in the Jaynes-Cummings model is responsible for interesting properties in the evolution of the micromaser where measuring atomic

2010 Mathematics Subject Classification: Primary: 81P15; Secondary: 46N50, 47N50, 81R30, $81 \mathrm{~S} 05$.

Key words and phrases: Wehrl entropy, atom-field system, Tavis-Cumming model, Q function. The paper is in final form and no version of it will be published elsewhere. 
properties one can infer information concerning the field. Such an analysis is also possible for TCM which can be considered as a tripartite quantum system. Quantum correlations have been studied in this entangled tripartite quantum system by Coffman et al. [7].

Ever since the mathematical formulation of Heisenberg uncertainty relation the standard deviation was considered to be the natural measure of uncertainty related to quantum fluctuations. Important effects in quantum optics such as squeezing and antibunching have been defined in terms of standard deviation. One can also formulate with the help of standard deviation measures of quantum uncertainty such as total noise or uncertainty radius, surface and volume. However, it has been observed that the entropy rather than the standard deviation is more reliable parameter to characterize the fluctuation of the field and nowadays this alternative approach based on the concept of entropy is studied extensively.

A good measure of purity of states is governed by Von Neumann quantum-mechanical entropy $1,1,2,3,8,9,10,11,12,15,16,17,18,19,23,24$, as it gives zero for all pure states and does not distinguish different types of pure states. On the other hand Wehrl defined a classical analogue of the Von Neumann entropy associated with a quantum state of the system which in terms of the Husimi $(Q)$ function can be written as

$$
S=-\frac{1}{\pi} \int Q(\alpha) \ln Q(\alpha) d^{2} \alpha .
$$

The definition above is justified as we know that Husimi $(Q)$ function does not take negative values for any $\alpha$. The definition of Wehrl entropy \begin{tabular}{|c|c|c|c|c|c|c|}
4,5 & 6 & 13 & 14 & 20 & 22 & shows the
\end{tabular} unique role played by coherent states $|\alpha\rangle$ as the $Q$ representation of the density operator is defined by

$$
Q(\alpha)=\langle\alpha|\rho| \alpha\rangle
$$

with the normalization condition

$$
\frac{1}{\pi} \int Q(\alpha) d^{2} \alpha=1
$$

In this paper we shall investigate the Wehrl entropy of the state generated by the interaction of the single mode of radiation field in a cavity which contains two two-level atoms (TCM) to understand the dynamical nature of the evolved TCM state. In the process we calculate analytically explicit value of the Wehrl entropy of the evolved state.

2. The state vector. We consider a single mode of quantized electromagnetic field interacting with an ensemble consisting of two two-level atoms. This model is known as Tavis-Cumming model or Dicke model. In rotating wave approximation the total Hamiltonian (assuming $\hbar=1$ ) describing the atom-field system is given by

$$
H=H_{0}+H_{1},
$$

where

$$
H_{0}=\omega_{e_{1}}\left|e_{1}\right\rangle\left\langle e_{1}\left|+\omega_{g_{1}}\right| g_{1}\right\rangle\left\langle g_{1}\left|+\omega_{e_{2}}\right| e_{2}\right\rangle\left\langle e_{2}\left|+\omega_{g_{2}}\right| g_{2}\right\rangle\left\langle g_{2}\right|+\gamma a^{\dagger} a \quad(\hbar=1),
$$

and

$$
H_{1}=\lambda_{1}\left(a\left|e_{1}\right\rangle\left\langle g_{1}\left|+a^{+}\right| g_{1}\right\rangle\left\langle e_{1}\right|\right)+\lambda_{2}\left(a\left|e_{2}\right\rangle\left\langle g_{2}\left|+a^{+}\right| g_{2}\right\rangle\left\langle e_{2}\right)\right.
$$




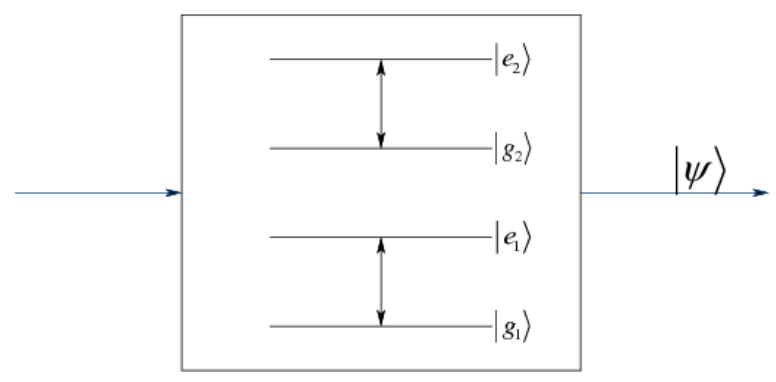

Fig. 1. Interaction of one mode field with two two-level atoms

Here $a^{\dagger}$ and $a$ are, respectively, the creation and annihilation operators for the field of frequency $\gamma \cdot|m\rangle\left(m=e_{1}, g_{1}, e_{2}, g_{2}\right)$ are the eigenstates of the atom with eigenfrequencies $\omega_{m}\left(m=e_{1}, g_{1}, e_{2}, g_{2}\right)$, and $\lambda_{r}(r=1,2)$ are the corresponding coupling constants. We assume the coupling constants $\lambda_{1}$ and $\lambda_{2}$ to be same and real throughout the paper. Then interaction Hamiltonian $H_{1}$ takes the form

$$
H_{1}=\lambda a\left(\left|e_{1}\right\rangle\left\langle g_{1}|+| e_{2}\right\rangle\left\langle g_{2}\right|\right)+\lambda a^{+}\left(\left|g_{1}\right\rangle\left\langle e_{1}|+| g_{2}\right\rangle\left\langle e_{2}\right|\right)
$$

The state vector of this atom-field coupling system at time $t$ can be described by $|\psi(t)\rangle=\sum_{n}\left(C_{e_{1} e_{2} n}\left|e_{1}, e_{2}, n\right\rangle+C_{e_{1} g_{2} n}\left|e_{1}, g_{2}, n\right\rangle+C_{g_{1} e_{2} n}\left|g_{1}, e_{2}, n\right\rangle+C_{g_{1} g_{2} n}\left|g_{1}, g_{2}, n\right\rangle\right)$

Substituting equation (4) in the Schrödinger equation we get

$$
j \frac{d}{d t}|\psi(t)\rangle=H_{1}|\psi(t)\rangle \text {. }
$$

Substituting (5) in (6) we get

$$
\begin{aligned}
i \dot{C}_{e_{1} e_{2} n} & =\lambda \sqrt{n+1}\left(C_{e_{1} g_{2} n+1}+C_{g_{1} e_{2} n+1}\right) \\
i \dot{C}_{e_{1} g_{2} n+1} & =\lambda \sqrt{n+1} C_{e_{1} e_{2} n}+\lambda \sqrt{n+2} C_{g_{1} g_{2} n+2} \\
i \dot{C}_{g_{1} e_{2} n+1} & =\lambda \sqrt{n+1} C_{e_{1} e_{2} n}+\lambda \sqrt{n+2} C_{g_{1} g_{2} n+2} \\
i \dot{C}_{g_{1} g_{2} n+1} & =\lambda \sqrt{n+1}\left(C_{g_{1} e_{2} n}+C_{e_{1} g_{2} n}\right)
\end{aligned}
$$

From (7), (8), (9) and (10) we now have

$$
C_{e_{1} g_{2} n+1}=C_{g_{1} e_{2} n+1}=d_{1} e^{i \lambda \sqrt{2(2 n+3)} t}+d_{2} e^{-i \lambda \sqrt{2(2 n+3)} t} .
$$

If the atoms are initially in the state $\left|\psi_{A}(0)\right\rangle$,

$$
\left|\psi_{A}(0)\right\rangle=\frac{1}{\sqrt{2}}\left(\left|e_{1} g_{2}\right\rangle+\left|g_{1} e_{2}\right\rangle\right)
$$

which means that the atoms are in the coherent superposition state of their eigenkets $\left|e_{1} g_{2}\right\rangle$ and $\left|g_{1} e_{2}\right\rangle$, and the field is in the superposition of the photon number states at time $t=0$

$$
\left|\psi_{f}(0)\right\rangle=\sum_{n} F_{n}|n\rangle
$$


where $\sum_{n}\left|F_{n}\right|^{2}=1$, then the state vector of the total system at $t=0$ can be described as

$$
|\psi(0)\rangle=\sum_{n}\left[\frac{1}{\sqrt{2}} F_{n}\left|e_{1}, g_{2}, n\right\rangle+\frac{1}{\sqrt{2}} F_{n}\left|g_{1}, e_{2}, n\right\rangle\right]
$$

Finally we get

$$
\begin{aligned}
C_{e_{1} e_{2} n}(t) & =\sqrt{\frac{n+1}{2 n+3}}(1-\cos (\lambda t \sqrt{4 n+6})) F_{n} \\
C_{e_{1} g_{2} n+1}(t) & =C_{g_{1} e_{2} n+1}(t)=\frac{i}{\sqrt{2}} \sin (\lambda t \sqrt{4 n+6}) F_{n} \\
C_{g_{1} g_{2} n+2}(t) & =\sqrt{\frac{n+2}{2 n+3}}(1-\cos (\lambda t \sqrt{4 n+6})) F_{n}
\end{aligned}
$$

where $F_{n}=e^{-|\alpha|^{2} / 2} \cdot \alpha^{n} / \sqrt{n !}$ for coherent field.

Substituting the values of $C_{e_{1} e_{2} n}(t), C_{e_{1} g_{2} n}(t), C_{g_{1} e_{2} n}(t)$ and $C_{g_{1} g_{2} n}$ from (15), (16) and (17) respectively in equation (5) we can obtain the state vector of the system at time $t$.

Now after the interaction with the field if we detect the atoms in the ground state $\left|g_{1} g_{2}\right\rangle$ after time $t_{1}$ then effectively atoms absorb no photons but project the cavity field into the state

$$
\begin{aligned}
\left|\psi\left(t_{1}\right)\right\rangle & =\frac{1}{\eta} \sum_{n=0}^{\infty} C_{g_{1} g_{2} n+2}\left(t_{1}\right)|n+2\rangle \\
& =\frac{1}{\eta} \sum_{n=0}^{\infty} \sqrt{\frac{n+2}{2 n+3}}\left(1-\cos \left(\lambda t_{1} \sqrt{4 n+6}\right)\right) F_{n}|n+2\rangle
\end{aligned}
$$

The atom collapses in the ground state $\left|g_{1} g_{2}\right\rangle$ with maximum fidelity

$$
\text { Fidelity }=\frac{\sum_{n=0}^{\infty} \frac{n+2}{2 n+3}(1-\cos (\lambda t \sqrt{4 n+6}))^{2}\left|F_{n}\right|^{2}}{\sum_{n=0}^{\infty}\left\{(1-\cos (\lambda t \sqrt{4 n+6}))^{2}+2 \sin ^{2}(\lambda t \sqrt{4 n+6})\right\}\left|F_{n}\right|^{2}}
$$

and we assume that the maximum fidelity occurs at $t=t_{1}$.

3. Husimi distribution and Wehrl entropy. To describe Wehrl entropy we need to first calculate Husimi $Q$ function

$$
\begin{aligned}
Q(\nu) & =\left|\left\langle\nu \mid \psi\left(t_{1}\right)\right\rangle\right|^{2} \\
& =\frac{e^{-|\alpha|^{2}-|\nu|^{2}}}{\eta_{1}^{2}}\left|\sum_{n=0}^{\infty} \sqrt{\frac{n+2}{2 n+3}}\left(1-\cos \left(\lambda t_{1} \sqrt{4 n+6}\right)\right) \frac{\bar{\nu}^{n+2} \alpha^{n}}{\sqrt{n !(n+2) !}}\right|^{2}
\end{aligned}
$$

where $\eta_{1}^{2}=\sum_{n=0}^{\infty}\left|C_{g_{1} g_{2} n+2}\left(t_{1}\right)\right|^{2}$. 
Now

$$
\begin{aligned}
Q(\nu) \sim & \frac{1}{\eta_{1}^{2}} e^{-|\alpha|^{2}-|\nu|^{2}}\left(\sum_{n=0}^{\infty} \frac{|\nu|^{n+2}|\alpha|^{n}}{\sqrt{n !(n+2) !}}\right)^{2} f\left(\alpha, \eta_{1}\right) \\
= & \frac{1}{\eta_{1}^{2}} e^{-|\alpha|^{2}-|\nu|^{2}}\left\{\sum_{n=0}^{\infty} \frac{|\nu|^{2 n+4}|\alpha|^{2 n}}{n !(n+2) !}\right. \\
& \left.+2 \sum_{m=n+1, n=0}^{\infty} \frac{|\nu|^{m+n+4}|\alpha|^{n+m}}{\sqrt{n ! m !(n+2) !(m+2) !}}\right\} f\left(\alpha, \eta_{1}\right) .
\end{aligned}
$$

Now we have

$$
\frac{1}{\pi} \int Q(\nu) d^{2} \nu=\frac{1}{\eta_{1}^{2}} f\left(\alpha, \eta_{1}\right) e^{-|\alpha|^{2}} \sum_{n, m=0}^{\infty} \frac{|\alpha|^{n+m}}{\sqrt{n ! m !(n+2) !(m+2) !}} \Gamma\left(\frac{n+m+6}{2}\right) .
$$

Let us now choose $f\left(\alpha, \eta_{1}\right)$ such that $\frac{1}{\pi} \int Q(\nu) d^{2} \nu=1$.

From 22 we now have

$$
f\left(\alpha, \eta_{1}\right)=\eta_{1}^{2} e^{|\alpha|^{2}}\left[\sum_{n, m=0}^{\infty} \frac{|\alpha|^{n+m}}{\sqrt{n ! m !(n+2) !(m+2) !}} \Gamma\left(\frac{n+m+6}{2}\right)\right]^{-1}
$$

Substituting the value of $f\left(\alpha, \eta_{1}\right)$ in 21 we get

$$
Q(\nu)=\chi \cdot e^{-|\nu|^{2}} \sum_{n, m=0}^{\infty} \frac{|\nu|^{n+m+4}|\alpha|^{n+m}}{\sqrt{n ! m !(n+2) !(m+2) !}}
$$

where

$$
\chi=\left[\sum_{n, m=0}^{\infty} \frac{|\alpha|^{n+m}}{\sqrt{n ! m !(n+2) !(m+2) !}} \Gamma\left(\frac{n+m+6}{2}\right)\right]^{-1} .
$$

We now plot Husimi $Q$ distribution for different values of square root of mean photon numbers $|\alpha|=1,3,5,7$ and observe the rapid change of the shape of the distribution as $|\alpha|$ changes.

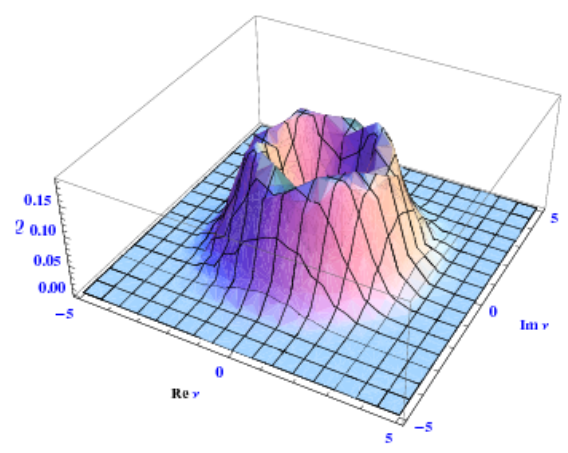

Fig. 2. Plot of $Q$ distribution for $|\alpha|=1$

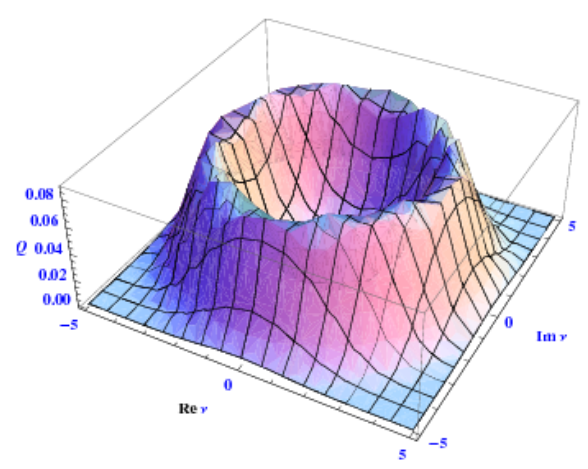

Fig. 3. Plot of $Q$ distribution for $|\alpha|=3$ 


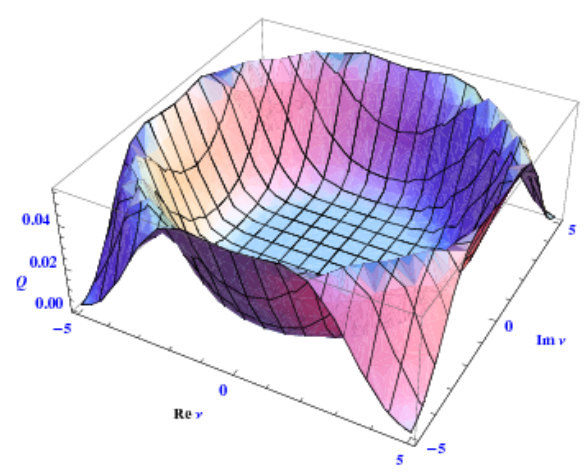

Fig. 4. Plot of $Q$ distribution for $|\alpha|=5$

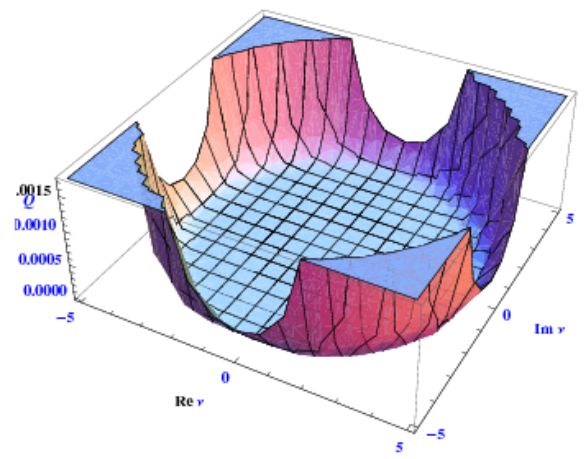

Fig. 5. Plot of $Q$ distribution for $|\alpha|=7$

Then

$$
\ln Q(\nu)=\ln \chi-\nu^{2}+\ln \sum_{n, m=0}^{\infty} \frac{|\nu|^{n+m+4}|\alpha|^{n+m}}{\sqrt{n ! m !(n+2) !(m+2) !}}
$$

Now

$$
\begin{aligned}
& Q(\nu) \ln Q(\nu)=\chi \ln \chi \cdot e^{-|\nu|^{2}} \sum_{n, m=0}^{\infty} \frac{|\nu|^{n+m+4}|\alpha|^{n+m}}{\sqrt{n ! m !(n+2) !(m+2) !}} \\
& -\chi \nu^{2} e^{-|\nu|^{2}} \sum_{n, m=0}^{\infty} \frac{|\nu|^{n+m+4}|\alpha|^{n+m}}{\sqrt{n ! m !(n+2) !(m+2) !}} \\
& +\chi e^{-|\nu|^{2}} \sum_{n, m=0}^{\infty} \frac{|\nu|^{n+m+4}|\alpha|^{n+m}}{\sqrt{n ! m !(n+2) !(m+2) !}} \cdot \ln \sum_{n, m=0}^{\infty} \frac{|\nu|^{n+m+4}|\alpha|^{n+m}}{\sqrt{n ! m !(n+2) !(m+2) !}}
\end{aligned}
$$

We now calculate Wehrl entropy of the field as follows:

From 26 we substitute the value of $Q(\nu) \ln Q(\nu)$ in the formula

$$
\begin{aligned}
S= & -\frac{1}{\pi} \int Q(\nu) \ln Q(\nu) d^{2} \nu \\
= & -\frac{\chi \ln \chi}{\pi} \cdot \int e^{-|\nu|^{2}} \sum_{n, m=0}^{\infty} \frac{|\nu|^{n+m+4}|\alpha|^{n+m}}{\sqrt{n ! m !(n+2) !(m+2) !}} d^{2} \nu \\
& +\frac{\chi}{\pi} \int \nu^{2} e^{-|\nu|^{2}} \sum_{n, m=0}^{\infty} \frac{|\nu|^{n+m+4}|\alpha|^{n+m}}{\sqrt{n ! m !(n+2) !(m+2) !}} d^{2} \nu \\
- & \frac{\chi}{\pi} \int e^{-|\nu|^{2}} \sum_{n, m=0}^{\infty} \frac{|\nu|^{n+m+4}|\alpha|^{n+m}}{\sqrt{n ! m !(n+2) !(m+2) !}} \\
& \times \ln \sum_{n, m=0}^{\infty} \frac{|\nu|^{n+m+4}|\alpha|^{n+m}}{\sqrt{n ! m !(n+2) !(m+2) !}} d^{2} \nu \\
= & I_{1}+I_{2}+I_{3} .
\end{aligned}
$$


Now, we put $\nu=r e^{i \theta}$ and $d^{2} \nu=r d r d \theta$ to get

$$
\begin{aligned}
I_{1} & =-\frac{\chi \ln \chi}{\pi} \int_{0}^{2 \pi} d \theta \int_{0}^{\infty} e^{-r^{2}} \sum_{n, m=0}^{\infty} \frac{r^{n+m+4}|\alpha|^{n+m}}{\sqrt{n ! m !(n+2) !(m+2) !}} r d r \\
& =-\chi \ln \chi \sum_{n, m=0}^{\infty} \frac{|\alpha|^{n+m}}{\sqrt{n ! m !(n+2) !(m+2) !}}, \Gamma\left(\frac{n+m+6}{2}\right)
\end{aligned}
$$

Similarly

$$
I_{2}=\chi \sum_{n, m=0}^{\infty} \frac{|\alpha|^{n+m}}{\sqrt{n ! m !(n+2) !(m+2) !}} \Gamma\left(\frac{n+m+8}{2}\right) .
$$

Again

$$
\begin{aligned}
I_{3}= & \frac{\chi}{\pi} \int e^{-|\nu|^{2}} \sum_{n, m=0}^{\infty} \frac{|\nu|^{n+m+4}|\alpha|^{n+m}}{\sqrt{n ! m !(n+2) !(m+2) !}} \\
& \times \ln \sum_{n, m=0}^{\infty} \frac{|\nu|^{n+m+4}|\alpha|^{n+m}}{\sqrt{n ! m !(n+2) !(m+2) !}} d^{2} \nu \\
= & -2 \chi \sum_{n, m=0}^{\infty} \frac{|\alpha|^{n+m}}{\sqrt{n ! m !(n+2) !(m+2) !}} \Gamma\left(\frac{n+m+6}{2}\right) \Psi\left(\frac{n+m+6}{2}\right) \\
& -2 a_{0} \chi \sum_{n, m=0}^{\infty} \frac{|\alpha|^{n+m}}{\sqrt{n ! m !(n+2) !(m+2) !}} \Gamma\left(\frac{n+m+6}{2}\right) \\
& -2 a_{1} \chi \sum_{n, m=0}^{\infty} \frac{|\alpha|^{n+m+1}}{\sqrt{n ! m !(n+2) !(m+2) !}} \Gamma\left(\frac{n+m+7}{2}\right) \\
& -2 a_{2} \chi \sum_{n, m=0}^{\infty} \frac{|\alpha|^{n+m+2}}{\sqrt{n ! m !(n+2) !(m+2) !}} \Gamma\left(\frac{n+m+8}{2}\right)+\ldots
\end{aligned}
$$

where the function $\Psi(x)$ is defined by

$$
\Psi(x)=\frac{\Gamma^{\prime}(x)}{\Gamma(x)}
$$

and the values of the coefficients $a_{i}$ are given in the following table:

\begin{tabular}{|c|c|}
\hline Coefficient & Numerical Value \\
\hline$a_{0}$ & -0.346574 \\
$a_{1}$ & 0.57735 \\
$a_{2}$ & 0.0374575 \\
$a_{3}$ & -0.000996473 \\
$a_{4}$ & -0.000240473 \\
$a_{5}$ & 0.0000196618 \\
$a_{6}$ & $2.68953 \times 10^{-6}$ \\
\hline
\end{tabular}

\begin{tabular}{|c|c|}
\hline Coefficient & Numerical Value \\
\hline$a_{7}$ & $-4.02432 \times 10^{-7}$ \\
$a_{8}$ & $-3.0295 \times 10^{-8}$ \\
$a_{9}$ & $8.10708 \times 10^{-9}$ \\
$a_{10}$ & $2.46262 \times 10^{-10}$ \\
$a_{11}$ & $-1.58362 \times 10^{-10}$ \\
$a_{12}$ & $1.29067 \times 10^{-12}$ \\
$\vdots$ & $\vdots$ \\
\hline
\end{tabular}


From (27), 28), 29) and (30) we get

$$
\begin{aligned}
S= & \ln \left(\sum_{n, m=0}^{\infty} \frac{|\alpha|^{n+m}}{\sqrt{n ! m !(n+2) !(m+2) !}} \Gamma\left(\frac{n+m+6}{2}\right)\right) \\
& +\chi \sum_{n, m=0}^{\infty} \frac{|\alpha|^{n+m}}{\sqrt{n ! m !(n+2) !(m+2) !}} \Gamma\left(\frac{n+m+8}{2}\right) \\
& -2 \chi \sum_{n, m=0}^{\infty} \frac{|\alpha|^{n+m}}{\sqrt{n ! m !(n+2) !(m+2) !}} \Gamma\left(\frac{n+m+6}{2}\right) \Psi\left(\frac{n+m+6}{2}\right) \\
& -2 a_{0} \\
& -2 a_{1} \chi \sum_{n, m=0}^{\infty} \frac{|\alpha|^{n+m+1}}{\sqrt{n ! m !(n+2) !(m+2) !}} \Gamma\left(\frac{n+m+7}{2}\right) \\
& -2 a_{2} \chi \sum_{n, m=0}^{\infty} \frac{|\alpha|^{n+m+2}}{\sqrt{n ! m !(n+2) !(m+2) !}} \Gamma\left(\frac{n+m+8}{2}\right)+\ldots
\end{aligned}
$$

We shall now plot Wehrl entropy with respect to the square root of mean photon number $|\alpha|$ of the initial field.

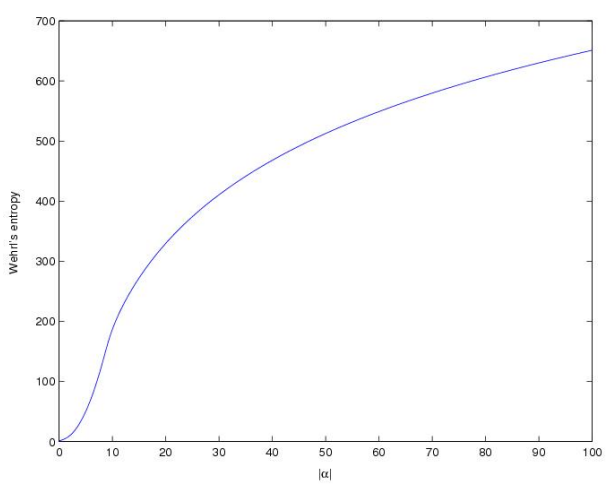

Fig. 6. Plot of Wehrl entropy neglecting the smaller coefficients of $a_{i}$

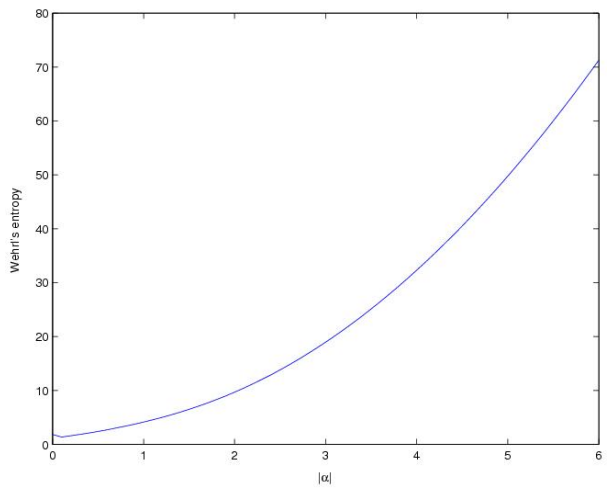

Fig. 7. Plot of Wehrl entropy for short range of $|\alpha|$

4. Conclusion. We have thus studied the dynamical evolution of Tavis-Cumming state and studied the fluctuation of the evolved state. Using the coherent states we have obtained different forms of Husimi distribution functions of the evolved TCM state explicitly for the values $|\alpha|=1,3,5,7$ and observe the rapid changes of the shape of the distribution as $|\alpha|$ increases. We have calculated Wehrl entropy analytically and plotted by neglecting smaller coefficients as well as for short range of square root of mean photon number. In the limit $|\alpha| \rightarrow 0$, the Wehrl entropy goes to 1.8475 . Wehrl entropy is useful to understand the intrinsic state fluctuation which in its turn generate some information theoretic measurement. 


\section{References}

[1] G. S. Agarwal, P. K. Phatak, Mesoscopic superposition of states with sub-Planck structures in phase space, Phys. Rev. A 70 (2004), 053813 (5 pp.).

[2] E. I. Aliskenderov, H. T. Dung, L. Knöll, Effects of atomic coherences in the JaynesCummings model: Photon statistics and entropy, Phys. Rev. A 48 (1993), 1604-1609.

[3] H. Araki, E. H. Lieb, Entropy inequalities, Commun. Math. Phys. 18 (1970), 160-170.

[4] V. Bužek, C. H. Keitel, P. L. Knight, Sampling entropies and operational phase-space measurement. I. General formalism, Phys. Rev. A 51 (1995), 2575-2593.

[5] V. Bužek, C. H. Keitel, P. L. Knight, Sampling entropies and operational phase-space measurement. II. Detection of quantum coherences, Phys. Rev. A 51 (1995), 2594-2601.

[6] V. Bužek, H. Moya-Cessa, P. L. Knight, Schrödinger-cat states in the resonant JaynesCummings model: Collapse and revival of oscillations of the photon-number distribution, Phys. Rev. A 45 (1992), 8190-8203.

[7] V. Coffman, J. Kundu, W. K. Wootters, Distributed entanglement, Phys. Rev. A 61 (2000), 052306 (5 pp.).

[8] C. Huang, L. Tang, F. Kong, J. Fang, M. Zhou, Entropy evolution of field interacting with V-type three-level atom via intensity-dependent coupling, Physica A 368 (2006), 25-30.

[9] W. K. Lai, V. Bužek, P. L. Knight, Dynamics of a three-level atom in a two-mode squeezed vacuum, Phys. Rev. A 44 (1991), 6043-6056.

[10] X. S. Li, C. D. Gong, Coherent properties of the stimulated emission from a three-level atom, Phys. Rev. A 33 (1986), 2801-2804.

[11] X. S. Li, Y. N. Peng, Quantum properties of a three-level atom interacting with two radiation fields, Phys. Rev. A 32 (1985), 1501-1514.

[12] X. Liu, Entropy behaviors and statistical properties of the field interacting with a $\Xi$-type three-level atom, Physica A 286 (2000), 588-598.

[13] A. Miranowicz, J. Bajer, M. R. B. Wahiddin, N. Imoto, Wehrl information entropy and phase distributions of Schrödinger cat and cat-like states, J. Phys. A 34 (2001), 3887-3896.

[14] A. Orłowski, Classical entropy of quantum states of light, Phys. Rev. A 48 (1993), 727-731.

[15] P. K. Pathak, G. S. Agarwal, Generation of a superposition of multiple mesoscopic states of radiation in a resonant cavity, Phys. Rev. A 71 (2005), 043823 (6 pp.).

[16] J. S. Peng, G. X. Li, A study on dissipation mechanism in two-photon laser, Acta Phys. Sinica 41 (1992), 1590-1597.

[17] J. S. Peng, G. X. Li, P. Zhou, Phase properties and atomic coherent trapping in the system of a three-level atom interacting with a bimodal field, Phys. Rev. A 46 (1992), 1516-1521.

[18] S. J. D. Phoenix, P. L. Knight, Fluctuations and entropy in models of quantum optical resonance, Ann. Phys. 186 (1988), 381-407.

[19] S. J. D. Phoenix, P. L. Knight, Comment on "Collapse and revival of the state vector in the Jaynes-Cummings model: An example of state preparation by a quantum apparatus", Phys. Rev. Lett. 66 (1991), 2833.

[20] S. J. D. Phoenix, P. L. Knight, Establishment of an entangled atom-field state in the Jaynes-Cummings model, Phys. Rev. A 44 (1991), 6023-6029.

[21] T. E. Tessier, I. H. Deutsch, A. Delgado, I. Fuentes-Guridi, Entanglement sharing in the two-atom Tavis-Cummings model, Phys. Rev. A 68 (2003), 062316 (10 pp.).

[22] A. Wehrl, General properties of entropy, Rev. Modern Physics 50 (1978), 221-260.

[23] H. I. Yoo, J. H. Eberly, Dynamical theory of an atom with two or three levels interacting with quantized cavity fields, Phys. Rep. 118 (1985), 239-337.

[24] S. B. Zhang, Generation of nonclassical states with a driven dispersive interaction, Phys. Rev. A 74 (2006), 043803 (5 pp.). 
